\title{
Article \\ Effect of Muscle-Specific Fatigue on the Risk of Anterior Cruciate Ligament Injury in Females
}

\author{
Jeheon Moon ${ }^{1}$, Jinseok Lee ${ }^{2}$, Keehyun Kim ${ }^{3}$, Dohoon Koo ${ }^{4}$, Jusung Lee ${ }^{5}$, Prabhat Pathak ${ }^{6}$, \\ Gustavo Adrian Ruiz Sanchez ${ }^{7}$ and Siddhartha Bikram Panday ${ }^{8, * \mathbb{D}}$ \\ 1 Department of Physical Education, Korea National University of Education, Chungbuk 28173, Korea; \\ jhmoon@knue.ac.kr \\ 2 Department of Sports Science, Korea Institute of Sport Science, Seoul 01794, Korea; js0420@kspo.or.kr \\ GK Networks, Seoul 06086, Korea; cookkee@gknetworks.kr \\ 4 Department of Exercise Prescription, College of Medical Science, Jeonju University, Jeonbuk 55069, Korea; \\ dohoonkoo@jj.ac.kr \\ 5 Department of Sport Science, Kangwon National University, Kangwon 24341, Korea; \\ jsleetime@kangwon.ac.kr \\ 6 Department of Physical Education, Seoul National University, Seoul 08826, Korea; prabhat@snu.ac.kr \\ 7 Department of Computer Engineering, Keimyung University, Daegu 42601, Korea; gustavo.ruiz@kmu.ac.kr \\ 8 Department of Sport and Leisure Studies, Keimyung University, Daegu 42601, Korea \\ * Correspondence: sid@kmu.ac.kr
}

Citation: Moon, J.; Lee, J.; Kim, K.; Koo, D.; Lee, J.; Pathak, P.;

Sanchez, G.A.R.; Panday, S.B. Effect of Muscle-Specific Fatigue on the Risk of Anterior Cruciate Ligament Injury in Females. Appl. Sci. 2021, 11, 4969 . https://doi.org/10.3390/ app11114969

Academic Editor: Redha Taiar

Received: 21 April 2021

Accepted: 25 May 2021

Published: 28 May 2021

Publisher's Note: MDPI stays neutral with regard to jurisdictional claims in published maps and institutional affiliations.

Copyright: (c) 2021 by the authors. Licensee MDPI, Basel, Switzerland. This article is an open access article distributed under the terms and conditions of the Creative Commons Attribution (CC BY) license (https:// creativecommons.org/licenses/by/ $4.0 /)$.

\begin{abstract}
The aim of our study was to investigate the effect of muscle-specific fatigue of the quadriceps and hamstring muscles on the biomechanical factors of anterior cruciate ligament (ACL) injury using musculoskeletal modeling techniques during directional diversion maneuver. Fifteen female subjects performed a directional diversion maneuver under three treatment conditions (quadriceps fatigue, hamstring fatigue, and control gait). Data from the 3D motion capture system and force platform were used to extract anterior/posterior ACL forces using the two-bundle ACL musculoskeletal modeling approach. A decrease in maximum extension (51.3\%) and flexion (50.7\%) torque after fatigue was observed. After quadriceps fatigue, the extension $(p=0.041)$ and adduction moments $(p=0.046)$ of the knee joint and the mean anterior bundle of ACL force $(p=0.021)$ decreased significantly. The knee flexion angle $(p=0.003)$, knee valgus angle $(p=0.013)$, and shear force $(p=0.043)$ decreased significantly after hamstring fatigue. The decrease in ACL force after quadriceps fatigue confirms its significant role in causing an ACL injury. However, no significant differences in ACL load after hamstring fatigue leads us to speculate that the antagonist muscle group, i.e., the hamstring, might not have a preventive mechanism against ACL injury.
\end{abstract}

Keywords: ACL; biomechanics; musculoskeletal modeling; running; knee kinematics

\section{Introduction}

Muscular fatigue is one of the major causes of anterior cruciate ligament (ACL) injury since fatigue adversely affects the somatosensory, proprioceptive, and muscular systems [1-3]. The effect is particularly prevalent during a complex task requiring a quick change in direction, such as directional diversion maneuver (DDM), which requires afferent feedback from these systems [4]. Hence, the adverse effects of fatigue on these systems may lead to a higher prevalence of ACL injury during DDM as it increases transitional and rotational forces exerted by the ACL due to instantaneous positioning and orientation of the lower limb $[5,6]$.

Studies report that approximately $70 \%$ of ACL injuries are non-contact in nature, with a three to four times higher prevalence among high school and collegiate age females than men competing in the same sports [7-9]. Various studies on walking, running, cutting, and landing have reported females exhibiting greater hip adduction and internal rotation with decreased knee flexion compared to male counterparts [10-14]. Hence, the effect of fatigue 
along with unexpected perturbations may lead to insufficient neuromuscular control and increase the risk of ACL injuries through increased knee valgus posture [15-17].

Biomechanical studies that analyzed postures with high ACL injury occurrence rates reported that a knee joint shear force (Knee shear ) of $2000 \mathrm{~N}$ and an adduction moment of $125 \mathrm{Nm}$ or more to be the injury threshold for ACL injury [18,19]. The quadriceps (QF) and the hamstring (HA) muscles are the two major muscle groups of the femur that affect the ACL load modulation; when fatigued, the risk of ACL injury is increased [20]. Numerous studies have been carried out to evaluate the effect of fatigue of the thigh muscles on ACL injury [21-23]. However, for most of these studies, fatigue was induced on thigh muscles around the femur as a whole, making it difficult to distinguish the exact nature of the muscle-specific relationship between QF and HA muscles with an ACL injury.

QF and HA muscle groups have a reciprocal function during ACL load modulation. Several cadaveric and musculoskeletal modeling studies have shown that a strong contraction of the QF results in an increase in the ACL load due to an increase in anterior shear force whereas, the co-contraction of the HA counteracts the anterior tibial shear force, decreasing the ACL load [24-26]. During the vertical jump task, the co-contraction of QF and HA has been reported to decrease peak anterior shear force by $292 \mathrm{~N}$ and have a protective effect on the ACL [27]. However, counter-arguments have been made about the role of HA in inhibiting the increase in ACL load. Simonsen, et al. [28] reported that HA does not play a role in ACL injury prevention due to its low activation during side-cutting maneuvers. Hence, understanding the role of each muscle on ACL load modulation after fatigue may help clinicians, trainers, and coaches create better ACL injury prevention protocols for female athletic populations.

Sporting activities involving rapid deceleration, such as planting instant during a DDM induce high impact and are significantly associated with different kinematics, kinetics, and muscle activation patterns compared to low-impact movement such as walking [29,30]. Despite a need to accurately replicate the forces and moments that act on the knee joint during high impact movement such as a DDM, there exist technical difficulties during measurements and the risk of injury to the participants. These limitations can be overcome by employing a musculoskeletal modeling (MSM) approach to estimate the ACL forces [31-36] directly. The MSM approach utilizes subject-specific anthropometrics, inverse kinematics (position) and inverse dynamics (forces and moment), computation of excitation of individual muscles, and forward dynamics to calculate ACL forces [29-31]. The physical characteristics of the muscles and tendons can be reflected in the MSM model to estimate the force-length-velocity relationship of the muscles effectively. Therefore, the purpose of this study was to investigate the effect of QF and HA fatigue on the ACL load using a musculoskeletal modeling approach. Hence, we examine the effect of kinematic and kinetic factors that lead to the changes in the ACL load due to muscle-specific fatigue in females. We hypothesize that the factors affecting ACL injury after QF fatigue will decrease compared to before $Q F$ fatigue and vice-versa in the case of HA fatigue.

\section{Materials and Methods}

\subsection{Participants}

A total of fifteen active female students enrolled in a sports science degree at a university (age: $21.9 \pm 1.79$ years; height: $166.5 \pm 6.35 \mathrm{~cm}$; body mass $(\mathrm{BM}): 61.5 \pm 7.51 \mathrm{~kg}$; body mass index: $22.2 \pm 2.27 \mathrm{~kg} / \mathrm{m}^{2}$ ) were recruited for the study. We used the sample size referring to previous musculoskeletal modeling studies [19,32,33]. During the recruitment, all participants were requested to complete the "modified Waterloo Footedness Questionnaire" which included the screening of the leg dominance (preference on kicking leg; right foot) and inclusion/exclusion criteria $[34,35]$. Particularly, the medical conditions such as any specific lower extremities muscular or joint injury for at least six months before the experiment such as ACL rupture, ACL reconstruction surgery, lower back pain, and medication conditions were collected. The self-reported physical activity participation level was reported to be more than three times per week for $2 \mathrm{~h}$ a day. Informed consent 
regarding the procedure and risks involved in the study was signed by the participants, which was approved by the Korea Institute of Sports Policy and Ethics Committee (IRB No. KISS-1810-044-01).

\subsection{Equipment}

All the experiments were carried out in the same biomechanical laboratory equipped with a temperature and humidity control system (PA030-A2ST-U; AR, KOR). A total of 18 infrared cameras (Qualisys, Oqus 7+, Gothenburg, Sweden) was used to collect the motion data at a sampling frequency of $200 \mathrm{~Hz}$. A force platform (Kistler Type 9287BA, Winterthur, Switzerland) was used to measure the three directional forces $\left(F_{X}, F_{Y}, F_{Z}\right)$ and moments $\left(M_{X}, M_{Y}, M_{Z}\right)$ at $1000 \mathrm{~Hz}$. All data were acquired using Qualisys Track Manager (ver.2.11, Gothenburg, Sweden) using an analog board for synchronization. An isokinetic strength measurement system (Cybex human norm, Ronkonkoma, NY, USA) was used to perform a knee flexion/extension task at a fixed angular velocity to induce targeted muscle fatigue (QF and $\mathrm{HA})$.

\subsection{Experimental Procedure}

The experimental protocol utilized to investigate muscle-specific fatigue on ACL load is presented in Figure 1. A pre-post study design was selected to assess the effect of treatment on selected parameters in the following order: (1) baseline measurement (pre$\mathrm{DDM}$ ); (2) treatment: muscle-specific fatigue protocol (QF or HA fatigue), general warm-up (control condition: $20 \mathrm{~min}$ walking); (3) post-measurement, i.e., after fatigue treatment (postDDM). Approximately 20 min was required to perform DDM after the fatigue treatment. Hence, we asked the participants to walk for 20-min to give a light intensity warm-up [36]. All participants underwent three experimental conditions and visited the laboratory three times. The order of the three treatments (QF or HA or control) was randomized, and the participants had at least a seven-day interval between each treatment.

On the first day, the participants got informed regarding the task, goal, process, and potential risks involved in the experiment. Then, the participants went through a pre-structured warm-up routine for $30 \mathrm{~min}$ which involved whole-body stretching [37], walking at preferred speed, performing five sprint runs on a straight pathway, and DDM runs at a preferred speed for familiarization of the task. Then, standard laboratory attire and shoes were provided to the participants. A total of 51 reflective markers were attached to 19 joints and 27 tracking markers to different segments (for the detailed location of markers: Supplementary Table S1) [38,39].

- Baseline measurement: Once the participants were ready, they performed three trials of the pre-DDM task, which involved running at a speed of $4.5 \pm 0.2 \mathrm{~m} / \mathrm{s}, 5 \mathrm{~m}$ behind the force platform, followed by a DDM on top of the force platform (Figure 2A). The trial was valid only when the subject's right foot gazed at the front and landed on the force plate and with a left turn at a range of $35^{\circ}-55^{\circ}$ in the direction of travel $[6,18,19,40]$. The running speed was controlled using 2 timing gates (Witty Microgate, Bolzano, Italy).

- Treatment: Muscle-specific fatigue treatment was performed using an isokinetic strength measurement system (Figure 2B) performing knee flexion/extension at a fixed angular velocity. Initially, the subject's reference value of the peak knee torque was established, taking the average peak torque value of 3 trials of knee flexion/extension at an angular velocity of $90 \%$ s. The reference value of the peak knee torque was determined before the pre-DDM task. The fatigue task of the QF muscle involved knee extension and flexion at a fixed angular velocity of $90 \% \mathrm{~s}$ and $300 \% \mathrm{~s}$, respectively, whereas the angular velocity for extension $(300 \%$ s) and flexion $(90 \%$ s) was reversed for the fatigue task of the HA muscle [22,41]. The onset of fatigue was set when the peak torque reached $50 \pm 2 \%$ three times consecutively, with a two-minute break between each trial [41,42]. Participants performed walking for $20 \mathrm{~min}$ at their preferred walking speed to avoid any muscle injury during the post DDM trials for the control condition. 
- Post measurement: During the muscle-specific fatigue protocol, the post-DDM task was performed immediately once the knee peak torque drop was confirmed. For the control treatment condition, participants performed a post-DDM task immediately after completing the 20-min walk, using the same protocol as the pre-test DDM task. Only the first successful trial was used for further analysis considering the fatigue recovery period.

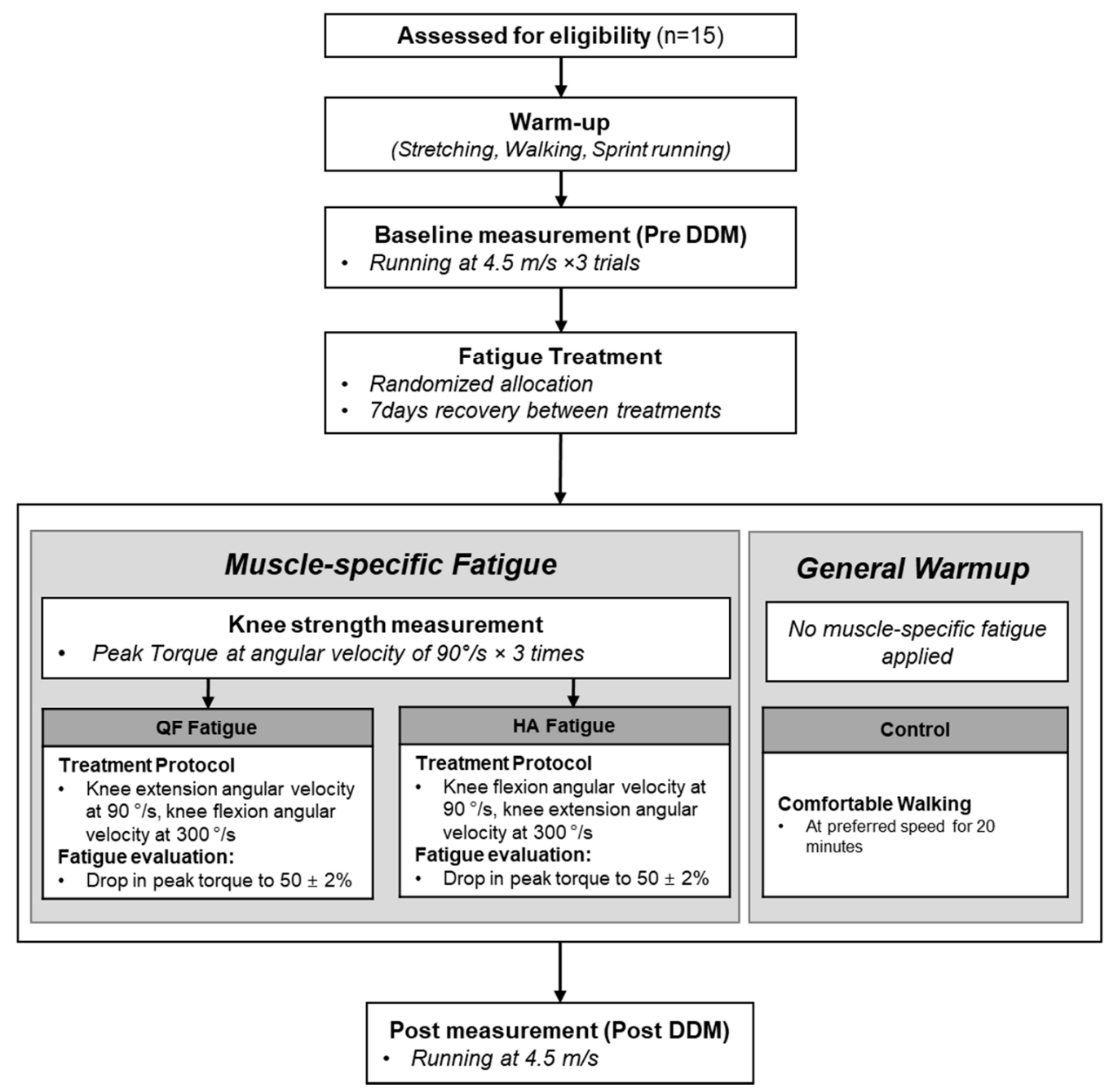

Figure 1. Experimental protocol. (1) Warm-up, (2) baseline measurement: running with DDM at $4.5 \mathrm{~m} / \mathrm{s}$, (3) treatment (muscle specific: (i) knee strength measurement; (ii) fatigue treatment; (iii) fatigue evaluation) (general fatigue: walking at preferred speed); (4) post-measurement: running with DDM at $4.5 \mathrm{~m} / \mathrm{s}$. The order of the test (control vs. quadriceps fatigue (QF) vs. hamstring (HA) fatigue) was randomized with at least 7 days interval between each condition.

\subsection{Data Analysis}

The raw signals obtained from the motion capture system and force platform were filtered using a zero-lag 4th order Butterworth filter with a cut-off frequency of $15 \mathrm{~Hz}$ using the visual 3D software (v5.01, C-Motion Inc., Rockville, MD, USA) [40]. Further, spline interpolation was used to fill missing sections in the dataset. 
(A)

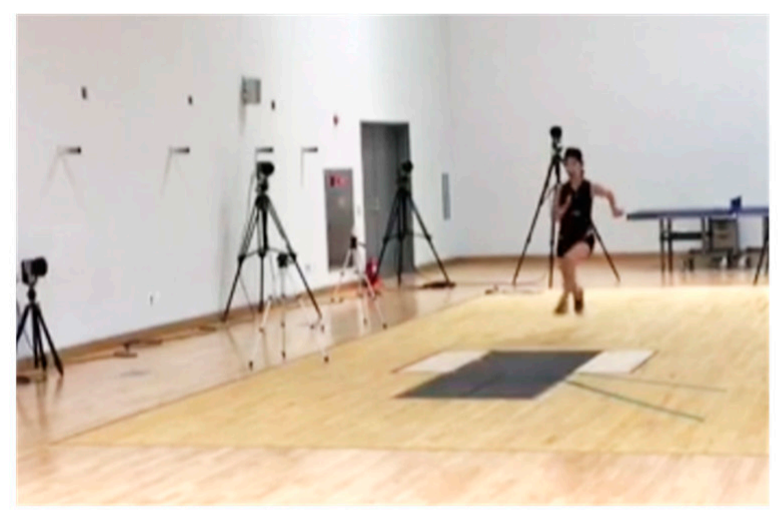

(C)

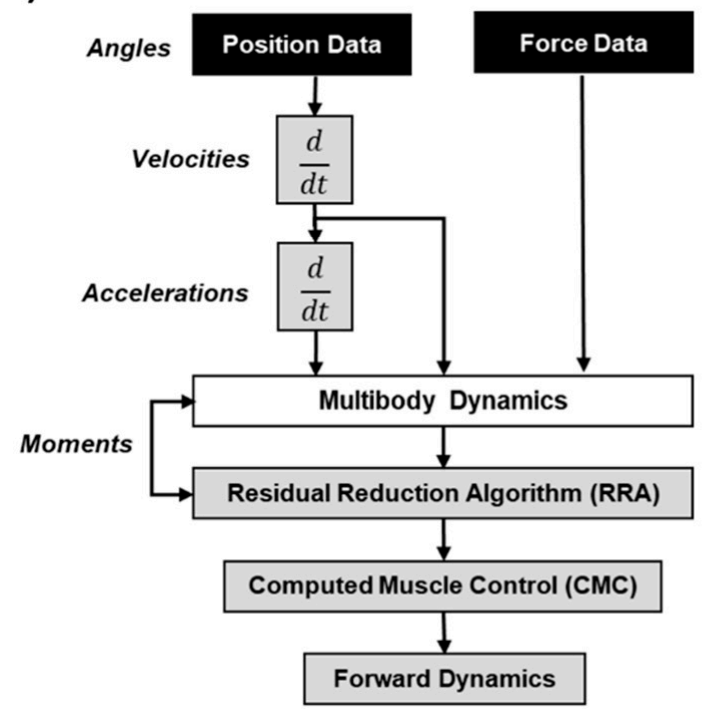

(B)

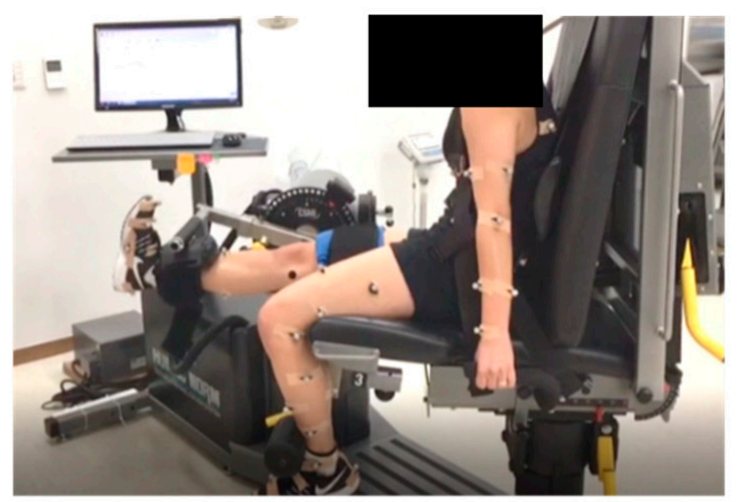

(D)

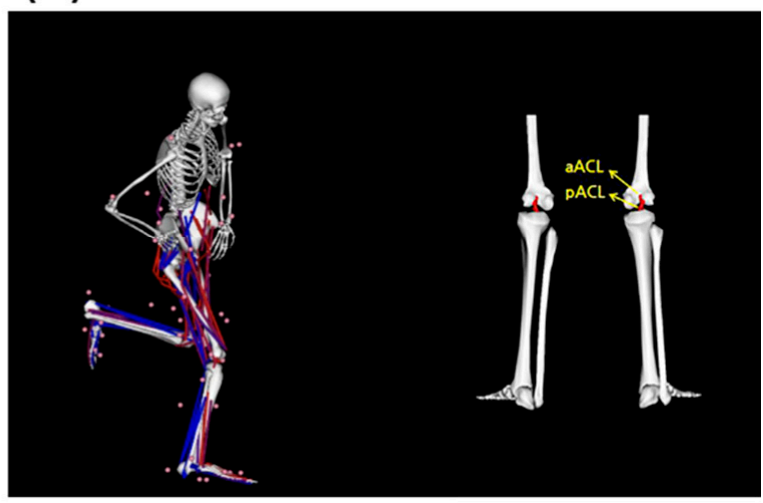

Figure 2. Experimental procedure. (A) Participants performing a direction diversion maneuver (DDM); (B) strength testing and fatigue treatment using an isokinetic dynamometer; (C) the process of calculating ACL load using a musculoskeletal modeling approach (MSM); (D) the MSM model used for the study. The open-source model provided in OpenSim (Stanford University, Stanford, CA, USA); two-bundle Anterior Cruciate Ligament (ACL) inserted between the femur and tibia (sagittal view).

\subsubsection{ACL Model}

The ACL load was calculated by inserting a two-bundle ACL model into an existing musculoskeletal model (Figure 2). A two-bundle ACL insertion is composed of 10 segments and has 23 degrees of freedom, with which a total of 92 muscles can be analyzed [43]. However, this model was limited to only a single degree of freedom; flexion/extension of the knee joint. We formulated a model that considered the flexion/extension, adduction/valgus, and the internal/external rotation of the knee joint to calculate the ACL load. In this study, the degree of freedom of the knee joint was increased (flexion/extension: $-120^{\circ}-+10^{\circ}$, adduction/valgus: $+15^{\circ}--15^{\circ}$, and internal/external rotation: $\left.+30^{\circ}--45^{\circ}\right)[30,44,45]$. The ACL load was calculated using the OpenSim software (version 3.3, Stanford University, Stanford, CA, USA).

\subsubsection{Calculation of Kinematic and Kinetic Variables}

The kinematic model was constructed based on the static standing calibration trial, and the joint center locations were estimated using the mid-point estimation method. The maximum and mean values for different parameters were computed using inverse 
kinematics and dynamics algorithms at the landing phase, i.e., when the subject's foot contacted the force plate during the DDM and was identified using the vertical direction force $\left(\mathrm{F}_{\mathrm{Z}}\right)$. The variables were also normalized with body mass and height for inter-subject comparability. The parameters computed for further analysis are as follows:

- Center of mass (COM): based on the location of COM of each segment expressed in meters (m); COM velocity is the first derivative of COM expressed in meters per second $(\mathrm{m} / \mathrm{s})$.

- Knee angle $\left(\right.$ knee $_{\text {ang }}\left({ }^{\circ}\right)$ ): refers to the flexion, valgus, and external rotation angles expressed in degrees $\left(^{\circ}\right)$.

- Knee joint force $(\mathrm{NN} / \mathrm{kg})$ : anterior tibial shear force $\left(\mathrm{knee}_{\text {shear }}\right)$, normalized shear force; normalized to body mass.

- $\quad$ Knee torque (knee ptorque) $(\mathrm{Nm})$ : mean and peak knee torque for flexion and extension expressed in newtons per meter.

- Knee moment $\left(\mathrm{knee}_{\mathrm{mom}}\right)(\mathrm{Nm}, \mathrm{Nm} /(\mathrm{kg} * \mathrm{HT}))$ : extension, adduction, internal rotation; All moments computed in the study are defined as internal moment and normalized with body height and mass.

- $\quad$ ACL forces $(\mathrm{N}, \mathrm{N} / \mathrm{kg}$ ): maximum anterior ACL (aACL) and posterior ACL (pACL) force, mean aACL and pACL force, and all forces were normalized to the body mass [31]. A detailed explanation of the steps involved to calculate the ACL load can be referred to in our previous study [30].

\subsection{Statistical Analysis}

All the data are presented as mean and standard deviation (SD). The normal distribution of data was determined using the Shapiro-Wilk test. The reliability of measurements was calculated using the Intraclass Correlation Coefficient (ICC) [46]. Two-way repeatedmeasures analysis of variance (ANOVA) was used for three fatigue conditions (QF muscle fatigue, HA muscle fatigue, control) and two measurement time points (pre-DDM and post-DDM) for each of the dependent variables $\left(\mathrm{COM}_{\text {velocity }}\right.$, knee $_{\text {angle }}$, knee $_{\text {force }}$, knee $_{\text {mom }}$, aACL, and pACL load). Bonferroni correction was performed as the post hoc test, and the statistical significance levels were set to $\alpha=0.05$. Effect sizes were calculated for the measured variables and interpreted according to Cohen: trivial (0-0.2), small (0.2-0.5), moderate (0.5-0.8), large (>0.8) [47]. All statistical analyses were performed using SPSS 23.0 (IBM, Chicago, IL, USA).

\section{Results}

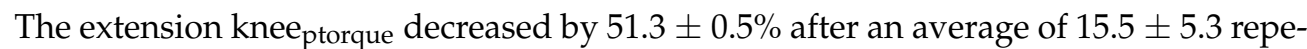
titions post-QF fatigue (pre-QF fatigue: $146.2 \pm 23.9 \mathrm{Nm}$; post-QF fatigue: $74.9 \pm 12.6 \mathrm{Nm}$ ).

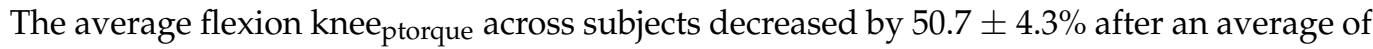
$18.9 \pm 4.0$ repetitions post-HA fatigue (pre-HA fatigue: $66.3 \pm 14.3 \mathrm{Nm}$; post-HA fatigue: $33.6 \pm 5.9 \mathrm{Nm}$ ). Hence, the fatigue condition performed in this study resulted in a decrease in peak knee torque within the range of $50.0 \pm 2.0 \%$. Therefore, an adequate amount of fatigue was induced (Table 1).

Table 1. Results of quadriceps (QF) and hamstring (HA) fatigue protocols.

\begin{tabular}{ccccc}
\hline & Pre (Nm) & Post $\mathbf{( N m )}$ & $\begin{array}{c}\text { Repetition } \\
\text { (Frequency) }\end{array}$ & Ratio (\%) \\
\hline QF fatigue & $146.2 \pm 23.9$ & $74.9 \pm 12.6$ & $15.5 \pm 5.3$ & $51.3 \pm 0.5$ \\
HA fatigue & $66.3 \pm 14.3$ & $33.6 \pm 5.9$ & $18.9 \pm 4.0$ & $50.7 \pm 4.3$ \\
\hline
\end{tabular}

In the force platform foot landing phase, $\mathrm{COM}_{\text {velocity }}$ according to the fatigue condition and the measurement time point was not statistically significant. There was no significant interaction effect, so it can be confirmed that the running speed was controlled throughout the experimental conditions (Supplementary Table S2). The flexion knee ${ }_{\text {angle }}$ significantly 
reduced in the case of the post-DDM for HA fatigue compared to post-DDM for QF fatigue; $\left(F_{(1,14)}=5.569 ; p=0.012\right)$ (Figure 3$)$. Further, the pairwise comparison showed that there was a significant reduction in flexion knee angle post-DDM task for HA fatigue condition; $\left(F_{(1,14)}=13.209 ; p=0.003\right)$. The interaction effect was observed due to a decrease in

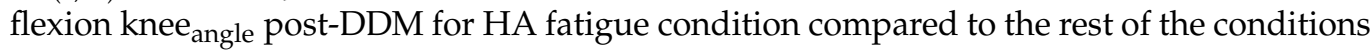
$\left(F_{(1,14)}=6.683 ; p=0.004 ; \mathrm{d}=0.938, \mathrm{ICC}=0.900\right)$. The pairwise comparison of the valgus knee $_{\text {angle }}$ also showed a significant reduction in the valgus knee angle post-DDM for HA fatigue condition $\left(F_{(1,14)}=8.024 ; p=0.013\right.$, ICC $\left.=0.958\right)$. However, no significant differences

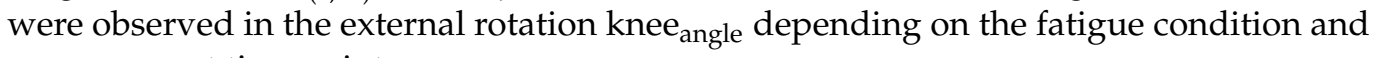
measurement time point.

(A)

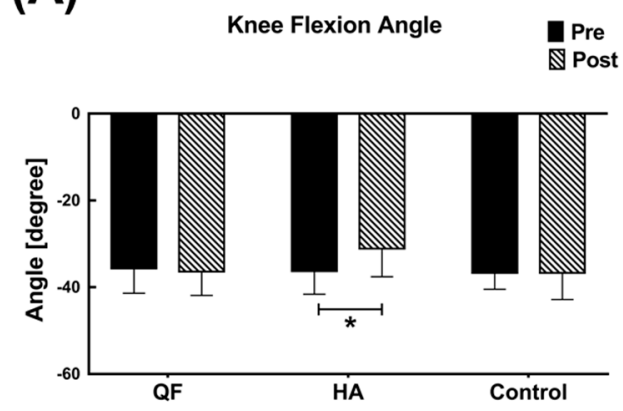

(C)

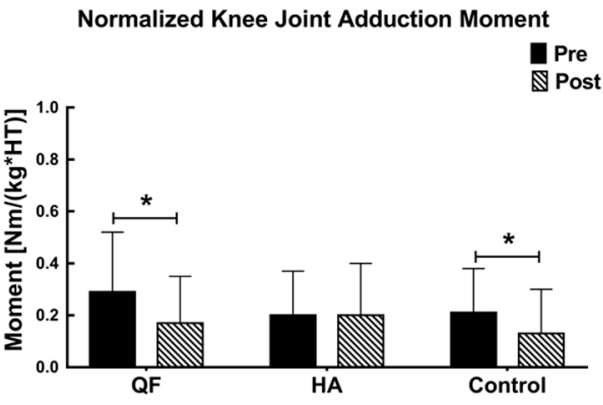

(E)

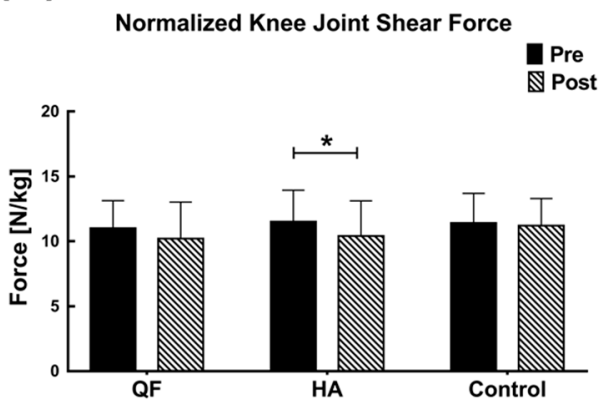

(B)

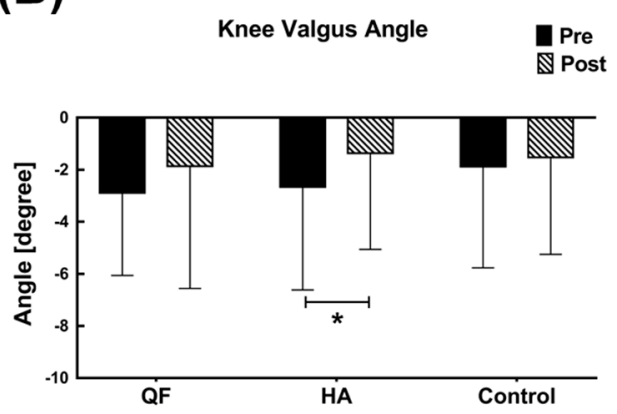

(D) Normalized Knee Joint Extension Moment

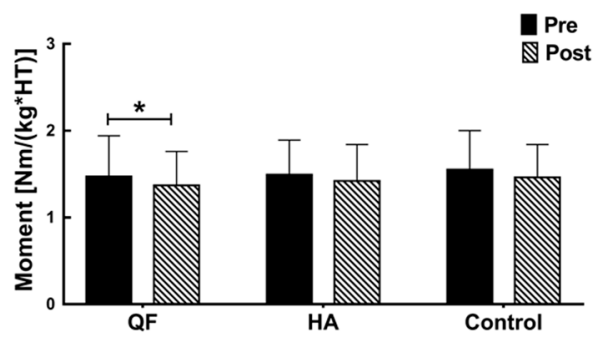

(F)

Normalized Mean Anterior ACL Force

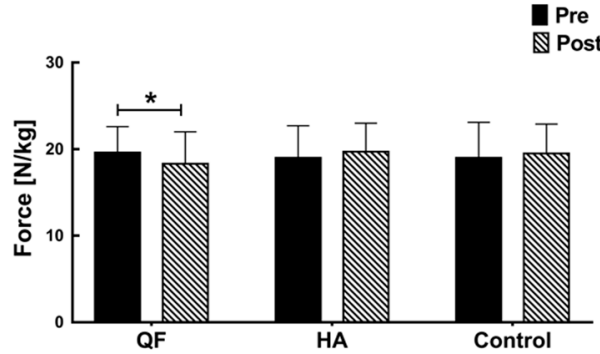

Figure 3. The mean and standard deviation of calculated variables. (A) Knee flexion angle; (B) knee valgus angle; (C) BM normalized knee joint adduction moment; (D) BM normalized knee joint extension moment; (E) BM normalized knee joint shear force; (F) normalized mean anterior ACL force. Pre-DDM condition in black, post-DDM in gray-dhaded. Significant differences between treatment conditions are denoted by asterisks (for detail, see Supplementary Table S2).

There was no significant interaction effect on the knee shear $_{\text {and }}$ the normalized knee $_{\text {shear }}$ based on the fatigue conditions and measurement time points. The pairwise 
comparison showed that there was a significant reduction in knee shear $_{(1,14)}=4.972$; $p=0.043, \mathrm{ICC}=0.907)$ and the BM normalized knee ${ }_{\text {shear }}\left(F_{(1,14)}=4.947 ; p=0.043\right.$, ICC $=0.906$ ) only in the case of the post-DDM for the HA fatigue condition compared to the pre-DDM. There was no significant interaction effect based on the fatigue condition and measurement time point for the extension, BM normalized extension, adduction, BM normalized adduction, internal rotation, and BM normalized internal rotation components

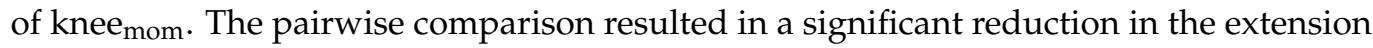
knee $_{\text {mom }}\left(F_{(1,14)}=5.603 ; p=0.033\right.$, ICC $\left.=0.905\right)$, BM normalized extension $\left(F_{(1,14)}=4.808\right.$; $p=0.046$, ICC $=0.902)$, adduction knee ${ }_{\text {mom }}\left(F_{(1,14)}=6.187 ; p=0.026, \mathrm{ICC}=0.916\right)$, and $\mathrm{BM}$ normalized adduction knee ${ }_{\mathrm{mom}}\left(F_{(1,14)}=5.995 ; p=0.028, \mathrm{ICC}=0.918\right)$, in the case of the post-DDM for the QF fatigue condition compared to the pre-DDM. Also, the pairwise comparison resulted in a significant reduction in adduction knee mom $\left(F_{(1,14)}=8.493\right.$; $p=0.011, \mathrm{ICC}=0.918)$ and BM normalized adduction knee mom $_{(1,14)}=8.360 ; p=0.012$, ICC $=0.918$ ) in the case of the post-DDM for the control condition compared to the preDDM task.

In the case of the ACL force, there was a significant interaction effect between fatigue condition and the measurement time point for the mean aACL force $\left(F_{(1,14)}=3.768\right.$; $p=0.045 ; \mathrm{d}=0.878, \mathrm{ICC}=0.858)$, and BM normalized mean pACL force $\left(F_{(1,14)}=3.410\right.$; $p=0.048 ; \mathrm{d}=0.174, \mathrm{ICC}=0.885)$. The pairwise comparison resulted in a significant reduction in the mean aACL force $\left(F_{(1,14)}=6.803 ; p=0.021, \mathrm{ICC}=0.858\right)$ and the BM normalized mean aACL force $\left(F_{(1,14)}=4.921 ; p=0.044\right.$, ICC $\left.=0.931\right)$ in the case of the post-DDM for the QF fatigue condition compared to the pre-DDM.

\section{Discussion}

The study aimed to evaluate the effect of muscle-specific fatigue of the QF and HA on the factors related to ACL injuries such as ACL load, knee angle, force, and moment during the DDM task. Therefore, we hypothesize that the factors affecting ACL injury after QF fatigue will decrease compared to before QF fatigue and vice-versa in the case of HA fatigue. It was confirmed that QF is the major muscle causing ACL injury since ACL load increased significantly after QF fatigue. However, the ACL load after HA fatigue did not significantly decrease, which leads us to speculate that HA might not have a preventive mechanism against ACL injury.

According to DeMorat, et al. [48], the ACL load varies according to the knee angle, with the highest load being observed at $30^{\circ}$ starting from a fully extended knee at $0^{\circ}$. Also, it was reported that the ACL load increases as the valgus and internal rotation of the knee joints increases. Particularly, studies conducted on women showed that the knee joints appear to be abducted up to $20^{\circ}$ in DDM and landing movements $[5,18]$. There was a significant reduction in the maximum flexion knee angle $_{\text {and knee valgus }}$ angle $_{\text {after }}$ HA fatigue in this study. An in-vivo study reported that modification to the tibiofemoral kinematics and its moment arm affects the activation of the hamstrings [49]. Additionally, in a closed kinematic chain, the decrease in the knee flexion angle also decreases the hip flexion angle. Since HA has a more significant mechanical advantage at the hip joint than at the knee joint, HA fatigue would decrease the hip and knee flexion angles [50]. This would result in the reduction of the muscle activation requirement on the HA muscle group. Further, a smaller moment arm of the HA at the knee would reduce torque, and the HA muscle group would not produce enough ACL shear forces at the knee joint to prevent ACL injury. Hence, it is considered that a direct relationship of change in knee kinematics, particularly during a dynamic activity such as DDM, may not be enough to confirm its effect on ACL load from this study.

During DDM, the shear force acting forward from the proximal direction of the tibia has been reported to cause ACL injury if the force exceeds $2000 \mathrm{~N}$ or more [26]. Numerous studies on the relationship between the contraction of QF and ACL load have reported that a strong contraction of QF in an extended knee position can increase the ACL load and the anterior tibial shear $[26,48]$ whereas, co-contraction of the HA was reported to suppress 
the increase in ACL load by offsetting the anterior tibial shear force [24,26,51]. However, the results of this study did not show statistically significant differences in the maximum knee $_{\text {shear }}$ in all three conditions (post-HA, post-QF, and control). Also, the maximum knee shear force was significantly lower after HA fatigue when comparing differences between pre and post DDM. Consequently, the mechanism by which HA reduces ACL load needs to be reconsidered since there was a reduction in knee $_{\text {shear }}$ after the fatiguing protocol in this study which contradicts the findings of previous studies; co-contraction between HA and QF results in a reduction of the knee shear $[24-26,28]$.

In order to evaluate the cause of ACL injuries, the moment acting on the knee joint must be considered [18,52]. Tsai et al. reported that the risk of ACL injury increases with an increase in the knee valgus angle, medial rotation angle, and adduction moment after fatigue during a squat jump [2]. Another study evaluating the effect of muscular fatigue on different movements by Zebis et al. reported a low activation of the QF and HA muscles with a gradual decrease in the activity of HA during DDM [23]. The study suggested that this reduction in HA activity after acute fatigue may increase the possibility of ACL injury during sporting situations. In this study, there was a reduction in the knee joint's maximum extension and adduction moment after QF fatigue. In contrast, no significant difference in knee moment was observed after HA fatigue. Hamstring activation has been known to provide a posterior force to protect the ACL damage when landing with a larger knee flexion angle [24]. However, when the hamstring is fatigued after repeated knee extension and acts as an antagonist, a decrease in the resultant joint moment has been reported [53]. This reduced activation of the hamstring with knee moment is considered to have a reduced effect on ACL loading. The results of these studies show similarities to this study, which did not show a substantial change in knee moment after hamstring fatigue.

The ACL load calculated during the DDM task in previous studies was limited to a 1-bundle ACL model $[30,44,45]$. Although another preceding study has used a two-bundle ACL model, the simulation was limited to evaluate the knee flexion and extension during gait and could be hardly used for an actual sporting condition [54]. Therefore, in this study, the load of aACL and pACL was calculated by inserting a two-bundle ACL model into the musculoskeletal modeling system, which was used to analyze directional diversion and landing maneuver $[21,45,54]$. In this study, maximum aACL and pACL force after QF and $\mathrm{HA}$ fatigue did not show significant changes. Although a significant reduction in the mean aACL after QF fatigue at the landing phase was observed, no significant changes in components of ACL force after HA fatigue were observed. Thus, although the hypothesis that the reduction in the ability of the neuromuscular system to produce force due to fatigue in QF could reduce ACL loads was indirectly proven, the hypothesis that fatigue of the HA muscle group would increase the ACL load could not be accepted. Therefore, the results of this study should be interpreted with caution, while more research is needed to reach certain conclusions. Nevertheless, we suggest coaches develop training methods that include strategies to reduce the eccentric contraction of quadriceps through knee flexion when performing movements with a sudden change in direction, such as DDM.

To our knowledge, no prior studies have analyzed the effect of muscle-specific fatigue of QF and HA muscle groups and its relation to the ACL injury on a dynamic motion such as DDM. Nevertheless, gait after QF fatigue has been reported to increase the knee external rotation angle and abduction moment [55]. Another study conducted on the effect of fatigue on lower extremities muscles during squat jump reported an increase in the knee joint's external and internal rotation angles and the adduction moment, which could increase the risk of ACL injury [2]. Although there are differences in the kinematics of the movement performed, these changes may attribute to the decrease in the neuromuscular ability of QF after fatigue, thereby reducing the factors related to increased ACL load such as extension moment and adduction moment of the knee joint.

There are three primary limitations associated with this investigation. First, the fatigue protocol utilized for the thigh muscles around the femur in this study involved only flexion/extension of the knee joint. Therefore, the relationship of factors associated with 
the ACL injury after fatiguing the rotational component of the femur (adduction, valgus, internal/external rotation) could not be identified. Further, future research can consider using the fatiguing protocol with the rotational treatment to observe its effect on the factors of an ACL injury. Secondly, we were not able to measure the muscle activity of QF and HA during DDM. The EMG sensor had to be attached before the fatigue condition, or else the delay in attachment could show a sign of recovery. However, as the isokinetic strength measurement system was used to induce fatigue in this study, the sensor could not be attached because subjects complained of pain at the sensor attachment site when strong pressure was applied. Therefore, if a fatigue-induced protocol that minimizes interference from the EMG sensor is developed in the future, it will increase our understanding of muscle activation and ACL load. Finally, the participants recruited in our study were recreationally active females. Further, research on female elite athletes from diverse sports backgrounds (strength-oriented-endurance) or levels of training experience could provide useful insight on the ACL injury mechanisms.

\section{Conclusions}

Although the ACL load after the HA fatigue did not show statistically significant differences, there was a significant reduction in ACL load after QF fatigue. This reduction of the neuromuscular system capacity of $Q F$ after fatigue reconfirms the previous findings that QF is the major muscle group causing ACL injuries by reducing the extension and adduction moment of the knee joint and thereby increasing the ACL load. Cautiously, we may indirectly deduce that HA might not have a preventive mechanism to reduce the risk of ACL injury even though there was a reduction in the knee valgus angle and anterior tibial shear force after HA fatigue. Hence, in our study, we indirectly confirmed the role of the QF and HA in increasing the risk of ACL injury using musculoskeletal modeling approaches.

Supplementary Materials: The following are available online at https://www.mdpi.com/article/ 10.3390/app11114969/s1, Table S1: Description of markers and location of attachment, Table S2. Results of Two way repeated measures ANOVA for kinematic and kinetic variables according to fatigue treatment.

Author Contributions: Conceptualization, J.M. and S.B.P.; Data curation, J.M., D.K. and J.L. (Jusung Lee); Formal analysis, J.M., J.L. (Jusung Lee) and G.A.R.S.; Funding acquisition, J.M.; Methodology, J.M., J.L. (Jinseok Lee), K.K. and S.B.P.; Project administration, J.M.; Software, D.K., J.L. (Jusung Lee), P.P. and G.A.R.S.; Validation, J.M., J.L. (Jinseok Lee), K.K., J.L. (Jusung Lee) and S.B.P.; Visualization, P.P. and S.B.P.; Writing—original draft, J.M., P.P. and S.B.P.; Writing—review and editing, J.M., J.L. (Jinseok Lee), K.K., D.K., J.L. (Jusung Lee), P.P., G.A.R.S. and S.B.P. All authors have read and agreed to the published version of the manuscript.

Funding: The research was supported by the Korea Institute of Sport Science and is part of the research project report (ISBN: 9788960111240) published in 2019. The funders had no role in study design, data collection and analysis, decision to publish, or preparation of the manuscript.

Institutional Review Board Statement: The study was conducted according to the guidelines of the Declaration of Helsinki, and approved by the Institutional Review Board (or Ethics Committee) of Korea Institute of Policy and Ethics Committee (IRB No. KISS -1806-034-01, date of acceptance: 22 June 2018).

Informed Consent Statement: Informed consent was obtained from all subjects involved in the study.

Data Availability Statement: Data available upon request.

Conflicts of Interest: The authors declare no conflict of interest.

\section{References}

1. Chappell, J.D.; Herman, D.C.; Knight, B.S.; Kirkendall, D.T.; Garrett, W.E.; Yu, B. Effect of fatigue on knee kinetics and kinematics in stop-jump tasks. Am. J. Sports Med. 2005, 33, 1022-1029. [CrossRef] 
2. Tsai, L.C.; Sigward, S.M.; Pollard, C.D.; Fletcher, M.J.; Powers, C.M. Effects of fatigue and recovery on knee mechanics during side-step cutting. Med. Sci. Sports Exerc. 2009, 41, 1952-1957. [CrossRef]

3. Enoka, R.M.; Stuart, D.G. Neurobiology of muscle fatigue. J. Appl. Physiol. 1992, 72, 1631-1648. [CrossRef] [PubMed]

4. Reed-Jones, R.J.; Vallis, L.A. Proprioceptive deficits of the lower limb following anterior cruciate ligament deficiency affect whole body steering control. Exp. Brain Res. 2007, 182, 249-260. [CrossRef]

5. Olsen, O.E.; Myklebust, G.; Engebretsen, L.; Bahr, R. Injury mechanisms for anterior cruciate ligament injuries in team handball: A systematic video analysis. Am. J. Sports Med. 2004, 32, 1002-1012. [CrossRef] [PubMed]

6. Weinhandl, J.T.; Earl-Boehm, J.E.; Ebersole, K.T.; Huddleston, W.E.; Armstrong, B.S.R.; O'Connor, K.M. Anticipatory effects on anterior cruciate ligament loading during sidestep cutting. Clin. Biomech. 2013, 28, 655-663. [CrossRef]

7. Joseph, A.M.; Collins, C.L.; Henke, N.M.; Yard, E.E.; Fields, S.K.; Comstock, R.D. A multisport epidemiologic comparison of anterior cruciate ligament injuries in high school athletics. J. Athl. Train. 2013, 48, 810-817. [CrossRef] [PubMed]

8. McNair, P.; Marshall, R.; Matheson, J. Important features associated with acute anterior cruciate ligament injury. N. Z. Med. J. 1990, 103, 537-539. [PubMed]

9. Agel, J.; Arendt, E.A.; Bershadsky, B. Anterior cruciate ligament injury in national collegiate athletic association basketball and soccer: A 13-year review. Am. J. Sports Med. 2005, 33, 524-531. [CrossRef]

10. Bien, D.P. Rationale and implementation of anterior cruciate ligament injury prevention warm-up programs in female athletes. J. Strength Cond. Res. 2011, 25, 271-285. [CrossRef]

11. Ford, K.R.; Myer, G.D.; Hewett, T.E. Valgus knee motion during landing in high school female and male basketball players. Med. Sci. Sports Exerc. 2003, 35, 1745-1750. [CrossRef]

12. Imwalle, L.E.; Myer, G.D.; Ford, K.R.; Hewett, T.E. Relationship between hip and knee kinematics in athletic women during cutting maneuvers: A possible link to noncontact anterior cruciate ligament injury and prevention. J. Strength Cond. Res. Natl. Strength Cond. Assoc. 2009, 23, 2223. [CrossRef]

13. Pollard, C.D.; Sigward, S.M.; Ota, S.; Langford, K.; Powers, C.M. The influence of in-season injury prevention training on lower-extremity kinematics during landing in female soccer players. Clin. J. Sport Med. 2006, 16, 223-227. [CrossRef]

14. Sigward, S.; Powers, C.M. The influence of experience on knee mechanics during side-step cutting in females. Clin. Biomech. 2006, 21, 740-747. [CrossRef] [PubMed]

15. Borotikar, B.S.; Newcomer, R.; Koppes, R.; McLean, S.G. Combined effects of fatigue and decision making on female lower limb landing postures: Central and peripheral contributions to ACL injury risk. Clin. Biomech. 2008, 23, 81-92. [CrossRef] [PubMed]

16. Myer, G.D.; Ford, K.R.; Brent, J.L.; Hewett, T.E. Differential neuromuscular training effects onACL injury risk factors in "high-risk" versus "low-risk" athletes. BMC Musculoskelet. Disord. 2007, 8, 1-7. [CrossRef]

17. Wojtys, E.M.; Huston, L.J.; Schock, H.J.; Boylan, J.P.; Ashton-Miller, J.A. Gender differences in muscular protection of the knee in torsion in size-matched athletes. JBJS 2003, 85, 782-789. [CrossRef] [PubMed]

18. McLean, S.G.; Huang, X.; Su, A.; Van Den Bogert, A.J. Sagittal plane biomechanics cannot injure the ACL during sidestep cutting. Clin. Biomech. 2004, 19, 828-838. [CrossRef]

19. McLean, S.G.; Huang, X.; van den Bogert, A.J. Investigating isolated neuromuscular control contributions to non-contact anterior cruciate ligament injury risk via computer simulation methods. Clin. Biomech. 2008, 23, 926-936. [CrossRef]

20. Behrens, M.; Mau-Moeller, A.; Wassermann, F.; Bruhn, S. Effect of fatigue on hamstring reflex responses and posterior-anterior tibial translation in men and women. PLoS ONE 2013, 8, e56988. [CrossRef]

21. Cortes, N.; Greska, E.; Kollock, R.; Ambegaonkar, J.; Onate, J.A. Changes in lower extremity biomechanics due to a short-term fatigue protocol. J. Athl. Train. 2013, 48, 306-313. [CrossRef]

22. Geiser, C.F.; O'Connor, K.M.; Earl, J.E. Effects of isolated hip abductor fatigue on frontal plane knee mechanics. Memed. Sci. Sports Exerc. 2010, 42, 535-545. [CrossRef] [PubMed]

23. Zebis, M.K.; Bencke, J.; Andersen, L.L.; Alkjaer, T.; Suetta, C.; Mortensen, P.; Kjaer, M.; Aagaard, P. Acute fatigue impairs neuromuscular activity of anterior cruciate ligament-agonist muscles in female team handball players. Scand. J. Med. Sci. 2011, 21, 833-840. [CrossRef] [PubMed]

24. Kernozek, T.W.; Ragan, R.J. Estimation of anterior cruciate ligament tension from inverse dynamics data and electromyography in females during drop landing. Clin. Biomech. 2008, 23, 1279-1286. [CrossRef] [PubMed]

25. Malinzak, R.A.; Colby, S.M.; Kirkendall, D.T.; Yu, B.; Garrett, W.E. A comparison of knee joint motion patterns between men and women in selected athletic tasks. Clin. Biomech. 2001, 16, 438-445. [CrossRef]

26. Shin, C.S.; Chaudhari, A.M.; Andriacchi, T.P. The influence of deceleration forces on ACL strain during single-leg landing: A simulation study. J. Biomech. 2007, 40, 1145-1152. [CrossRef] [PubMed]

27. Navacchia, A.; Ueno, R.; Ford, K.R.; DiCesare, C.A.; Myer, G.D.; Hewett, T.E. EMG-Informed Musculoskeletal Modeling to Estimate Realistic Knee Anterior Shear Force During Drop Vertical Jump in Female Athletes. Ann. Biomed. Eng. 2019, 47, 2416-2430. [CrossRef]

28. Simonsen, E.B.; Magnusson, S.P.; Bencke, J.; Naesborg, H.; Havkrog, M.; Ebstrup, J.F.; Sørensen, H. Can the hamstring muscles protect the anterior cruciate ligament during a side-cutting maneuver. Scand. J. Med. Sci. 2000, 10, 78-84. [CrossRef]

29. Lee, J.; Pathak, P.; Panday, S.B.; Moon, J. Effect of Foot-Planting Strategy on Anterior Cruciate Ligament Loading in Women During a Direction Diversion Maneuver: A Musculoskeletal Modeling Approach. Orthop. J. Sports Med. 2020, 8, 2325967120963180. [CrossRef] 
30. Moon, J.; Kim, H.; Lee, J.; Panday, S.B. Effect of wearing a knee brace or sleeve on the knee joint and anterior cruciate ligament force during drop jumps: A clinical intervention study. Knee 2018, 25, 1009-1015. [CrossRef]

31. Delp, S.L.; Anderson, F.C.; Arnold, A.S.; Loan, P.; Habib, A.; John, C.T.; Guendelman, E.; Thelen, D.G. OpenSim: Open-source software to create and analyze dynamic simulations of movement. IEEE Trans. Biomed. Eng. 2007, 54, 1940-1950. [CrossRef] [PubMed]

32. Donnelly, C.J.; Chinnasee, C.; Weir, G.; Sasimontonkul, S.; Alderson, J. Joint dynamics of rear- and fore-foot unplanned sidestepping. J. Sci. Med. Sport 2017, 20, 32-37. [CrossRef] [PubMed]

33. Chaudhari, A.M.; Hearn, B.K.; Andriacchi, T.P. Sport-dependent variations in arm position during single-limb landing influence knee loading: Implications for anterior cruciate ligament injury. Am. J. Sports Med. 2005, 33, 824-830. [CrossRef]

34. Elias, L.J.; Bryden, M.P.; Bulman-Fleming, M.B. Footedness is a better predictor than is handedness of emotional lateralization. Neuropsychologia 1998, 36, 37-43. [CrossRef]

35. Van Melick, N.; Meddeler, B.M.; Hoogeboom, T.J.; Nijhuis-van der Sanden, M.W.; van Cingel, R.E. How to determine leg dominance: The agreement between self-reported and observed performance in healthy adults. PLoS ONE 2017, 12, e0189876. [CrossRef] [PubMed]

36. Law, R.Y.; Herbert, R.D. Warm-up reduces delayed-onset muscle soreness but cool-down does not: A randomised controlled trial. Aust. J. Physiother. 2007, 53, 91-95. [CrossRef]

37. Garber, C.E.; Blissmer, B.; Deschenes, M.R.; Franklin, B.A.; Lamonte, M.J.; Lee, I.-M.; Nieman, D.C.; Swain, D.P. Quantity and quality of exercise for developing and maintaining cardiorespiratory, musculoskeletal, and neuromotor fitness in apparently healthy adults: Guidance for prescribing exercise. Med. Sci. Sports Exerc. 2011. [CrossRef] [PubMed]

38. Collins, T.D.; Ghoussayni, S.N.; Ewins, D.J.; Kent, J.A. A six degrees-of-freedom marker set for gait analysis: Repeatability and comparison with a modified Helen Hayes set. Gait. Posture 2009, 30, 173-180. [CrossRef] [PubMed]

39. Wu, G.; Siegler, S.; Allard, P.; Kirtley, C.; Leardini, A.; Rosenbaum, D.; Whittle, M.; D'Lima, D.D.; Cristofolini, M.; Witte, H.; et al. ISB recommendation on definitions of joint coordinate system of various joints for the reporting of human joint motion-part I: Ankle, hip, and spine. J. Biomech. 2002, 35, 543-548. [CrossRef]

40. Donnelly, C.J.; Lloyd, D.G.; Elliott, B.C.; Reinbolt, J.A. Optimizing whole-body kinematics to minimize valgus knee loading during sidestepping: Implications for ACL injury risk. J. Biomech. 2012, 45, 1491-1497. [CrossRef] [PubMed]

41. Hantes, M.E.; Tsarouhas, A.; Giakas, G.; Spiropoulos, G.; Sideris, V.; Christel, P.; Malizos, K.N. Effect of fatigue on tibial rotation after single- and double-bundle anterior cruciate ligament reconstruction: A 3-dimensional kinematic and kinetic matched-group analysis. Am. J. Sports Med. 2012, 40, 2045-2051. [CrossRef]

42. Bellew, J.W.; Fenter, P.C. Control of balance differs after knee or ankle fatigue in older women. Arch. Phys. Med. Rehabil. 2006, 87, 1486-1489. [CrossRef] [PubMed]

43. Dorn, T.W.; Lin, Y.-C.; Pandy, M.G. Estimates of muscle function in human gait depend on how foot-ground contact is modelled. Comput. Methods Biomech. Biomed. Engin. 2012, 15, 657-668. [CrossRef] [PubMed]

44. Kar, J.; Quesada, P.M. A numerical simulation approach to studying anterior cruciate ligament strains and internal forces among young recreational women performing valgus inducing stop-jump activities. Ann. Biomed. Eng. 2012, 40, 1679-1691. [CrossRef]

45. Kar, J.; Quesada, P.M. A musculoskeletal modeling approach for estimating anterior cruciate ligament strains and knee anteriorposterior shear forces in stop-jumps performed by young recreational female athletes. Ann. Biomed. Eng. 2013, 41, 338-348. [CrossRef] [PubMed]

46. Hopkins, W.; Marshall, S.; Batterham, A.; Hanin, J. Progressive statistics for studies in sports medicine and exercise science. Med. Sci. Sports Exerc. 2009, 41, 3. [CrossRef] [PubMed]

47. Cohen, J. Statistical Power Analysis for the Behavioral Sciences; Academic Press: New York, NY, USA; London, UK, 1988.

48. DeMorat, G.; Weinhold, P.; Blackburn, T.; Chudik, S.; Garrett, W. Aggressive quadriceps loading can induce noncontact anterior cruciate ligament injury. Am. J. Sports Med. 2004, 32, 477-483. [CrossRef]

49. Navacchia, A.; Kefala, V.; Shelburne, K.B. Dependence of Muscle Moment Arms on In Vivo Three-Dimensional Kinematics of the Knee. Ann. Biomed. Eng. 2017, 45, 789-798. [CrossRef]

50. Kellis, E.; Galanis, N.; Kofotolis, N.; Hatzi, A. Effects of hip flexion angle on surface electromyographic activity of the biceps femoris and semitendinosus during isokinetic knee flexion. Muscles Ligaments Tendons J. 2017, 7, 286. [CrossRef]

51. Navacchia, A.; Bates, N.A.; Schilaty, N.D.; Krych, A.J.; Hewett, T.E. Knee abduction and internal rotation moments increase ACL force during landing through the posterior slope of the tibia. J. Orthop. Res. 2019, 37, 1730-1742. [CrossRef]

52. Markolf, K.L.; Burchfield, D.M.; Shapiro, M.M.; Shepard, M.F.; Finerman, G.A.; Slauterbeck, J.L. Combined knee loading states that generate high anterior cruciate ligament forces. J. Orthop. Res. 1995, 13, 930-935. [CrossRef] [PubMed]

53. Kellis, E. Antagonist moment of force during maximal knee extension in pubertal boys: Effects of quadriceps fatigue. Eur. J. Appl. Physiol. 2003, 89, 271-280. [CrossRef] [PubMed]

54. Xu, H.; Bloswick, D.; Merryweather, A. An improved OpenSim gait model with multiple degrees of freedom knee joint and knee ligaments. Comput. Methods Biomech. Biomed. Eng. 2015, 18, 1217-1224. [CrossRef] [PubMed]

55. Murdock, G.H.; Hubley-Kozey, C.L. Effect of a high intensity quadriceps fatigue protocol on knee joint mechanics and muscle activation during gait in young adults. Eur. J. Appl. Physiol. 2012, 112, 439-449. [CrossRef] [PubMed] 